\title{
On Outage Analysis in Overlay CCRN With RF Energy Harvesting and Co-Channel Interference
}

\section{SUTANU GHOSH ( $\nabla$ sutanuresearchiiests@gmail.com )}

Indian Institute of Engineering Science and Technology https://orcid.org/0000-0001-5660-7109

\section{Santi P. Maity}

Indian Institute of Engineering Science and Technology

\section{Tamaghna Acharya}

Indian Institute of Engineering Science and Technology

\section{Research Article}

Keywords: SWIPT, spectrum-sharing, co-channel interference, cooperative cognitive radio network

Posted Date: December 28th, 2021

DOI: https://doi.org/10.21203/rs.3.rs-1145636/v1

License: (1) This work is licensed under a Creative Commons Attribution 4.0 International License.

Read Full License 


\title{
On Outage Analysis in Overlay CCRN with RF Energy Harvesting and Co-channel Interference
}

\author{
Sutanu Ghosh - Santi P. Maity - Tamaghna Acharya
}

the date of receipt and acceptance should be inserted later

\begin{abstract}
This paper explores the impact of co-channel interference (CCI) on the link outage and radio frequency $(\mathrm{RF})$ energy harvesting $(\mathrm{EH})$. For analysis, cooperative cognitive radio network (CCRN) architecture is considered as system model that supports one-way primary user (PU) and two-way secondary user (SU) communications, using an overlay mode of spectrum sharing. Closed form outage expressions are derived for both $\mathrm{PU}$ and SU network in presence of multiple antennas at PUs and CCI at SUs. The effect of CCI on the system performance is studied with respect to interference-to-noise-ratio (INR), transmission power, number of antennas and number of CCI sources. Performance gains are found to achieve $\sim 20 \%$ and $\sim 15 \%$ for PU and SU outage in two antenna system over a single antenna one.
\end{abstract}

Keywords SWIPT, spectrum-sharing, co-channel interference, cooperative cognitive radio network.

\section{Introduction}

Despite the tremendous advancements over the last few decades, wireless networks still face various challenges that include spectrum scarcity, coverage area, reliability in data transmission, co-channel interference (CCI), sustainable network operation etc. Co-operative cognitive radio networks (CCRNs) [1] have been emerging as a prominent network architecture for addressing some of the above challenges particularly spectrum scarcity

\section{Authors Address}

Indian Institute of Engineering Science and Technology,

Shibpur, Howrah-711103, India, E-mail: sutanuresearchiiests@gmail.com; tamaghna.acharya@ieee.org; santipmaity@it.iiests.ac.in. and sustainable network operation, leveraging the advancements in simultaneous wireless information and power transfer (SWIPT) [2] and cognitive radio enabled spectrum sharing communication [3]. Two-way relay based overlay mode of CCRN can be designed that provides two simultaneous communication sessions through the sharing of primary user (PU) spectrum by secondary users (SUs). The model shows potential applications in internet-of-things (IoT) driven by deviceto-device (D2D) based massive machine-type communications (mMTC) over cellular spectrum [4]. It is seen that CCI has a dual impact on SWIPT enabled relaying. It appears in the form of interference, leading to a limitation on the data transmission rate of the relay. At the same time, being a radio frequency ( $\mathrm{RF}$ ) signal, can purposely be used as a source of energy harvesting $(\mathrm{EH})$ and thereby provides relaying power. In the recent literature, decode-and-forward (DF) relay assisted network performance is studied for RF-EH enabled one-way unlicensed SU communication by considering both potential and adverse effect of interference [5]-[7]. In [5], the SU outage probability and ergodic capacity are studied together for both delay-limited and delay-tolerant transmission modes using an underlay mode of CCRN. The dual effect of CCI is also investigated in [6] to analyse the performance of IoT communication over cellular networks using power splitting (PS) and time switching (TS) protocols. In [7], the authors consider the impact of CCI on SWIPT enabled communication using overlay mode of spectrum sharing. Three separate phases are considered in [7] for one-way PU communication and one-way SU communication purpose.

To the best of our knowledge, none of the aforementioned studies have investigated the scope of SWIPT enabled two-way reliable SU communication. Further, multi-antenna transmission, which is a key feature of 
modern cellular communication (i,e, LTE, 5G) has not also been explored in above studies. Besides, the usage of dedicated time slot for $\mathrm{EH}$ is found to be inefficient technique [8]. Instead, transmission of energy and information at same phase using hybrid power-time switching relaying (HPTSR) protocol [9] is beneficial to achieve high throughput. In addition, for effective RF-EH, Nakagami fading model is encouraged in the literature [10]. Motivated by all these facts together, the present work investigates the impact of CCI on the reliability of a multi-antenna, spectrum sharing communication over Nakagami fading channel in a SWIPT enabled CCRN framework. We consider HPTSR protocol for SWIPT operation. Moreover, unlike [7], a more realistic system architecture with compact frame structure is adopted in this study to achieve better reliability for one-way $\mathrm{PU}$ and two-way SU communications with RF-EH.

\section{System Model and Protocol Description}

Fig. 1 shows a CCRN system that enables one-way communication between $\mathrm{PU}$ nodes $\left(\mathrm{PU}_{T}\right.$ and $\left.\mathrm{PU}_{R}\right)$ and two-way communications between pair of SU nodes $\left(\mathrm{SU}_{1}\right.$ and $\mathrm{SU}_{2}$ ). Due to some obstacles between $\mathrm{PU}$ nodes (absence of line-of-sight), $\mathrm{PU}_{T}$ needs some relaying help, nearby SU nodes may forward this data transmission towards $\mathrm{PU}_{R}$. In return, $\mathrm{SU}$ can access the licensed spectrum of PU network for its own data communication. $\mathrm{PU}_{T}$ is assumed to be equipped with multiple antennas while $\mathrm{SU}$ nodes and $\mathrm{PU}_{R}$ have single antenna in each. It is also assumed that $\mathrm{PU}_{T}$ node in the network can have scope of availing sufficient energy backup while the SU nodes are energized only by $\mathrm{EH}$ from RF signals using HPTSR protocol. All the links between the pair of nodes follow Nakagami distribution. PU and SU target rate of data transmission are set as $\mathscr{R}_{p}$ and $\mathscr{R}_{s}$, respectively.

Let $\mathbf{h}_{1 j, n}=\left[h_{1 j, 1}, h_{1 j, 2}, \ldots, h_{1 j, N_{p_{a}}}\right], h_{2 j}$ and $h_{s}(\mathrm{j}$ $\in 1,2)$ be the channel coefficients between $\mathrm{PU}_{T}$ and $\mathrm{SU}_{j}, \mathrm{SU}_{j}$ and $\mathrm{PU}_{R}, \mathrm{SU}_{1}$ and $\mathrm{SU}_{2}$, respectively, where $\mathbf{h}_{1 j, n} \sim \mathcal{C N}\left(m_{k}, 1\right)$ and $h_{2 j}, h_{s} \sim \mathcal{C N}\left(m_{k}, 1\right)$. $\zeta_{j i}$ is the channel coefficient between the $\mathrm{SU}_{j}$ and the $\mathrm{i}^{\text {th }}$ interferer $\left(\mathrm{I}_{i}\right)$, with $\zeta_{j i} \sim \mathcal{C N}\left(m_{k}, 1\right)$. Symbol $\mathrm{m}_{k}$ indicates the Nakagami shaping factor and $D s j 1, D s j 2$, $D_{3}$ and $d_{j i}$ are the distances between $\mathrm{PU}_{T}-\mathrm{SU}_{j}, \mathrm{SU}_{j}$ $\mathrm{PU}_{R}, \mathrm{SU}_{1}-\mathrm{SU}_{2}$ and $\mathrm{SU}_{j}-\mathrm{I}_{i}$, respectively. $n_{p u}=n_{s u} \sim$ $\mathcal{C N}\left(0, \sigma^{2}\right)$ indicate the additive white Gaussian noise (AWGN) at the receiver of $\mathrm{PU}$ and SU nodes and $\sigma^{2}$ indicates AWGN variance. Necessary channel state information is assumed to be unavailable at PU nodes.

In the first phase of transmission frame, $\mathrm{PU}_{T}$ sends its signal $X_{p t}$ to both the $\mathrm{SU}$ nodes. $\mathrm{SU}_{1}$ and $\mathrm{SU}_{2}$ use

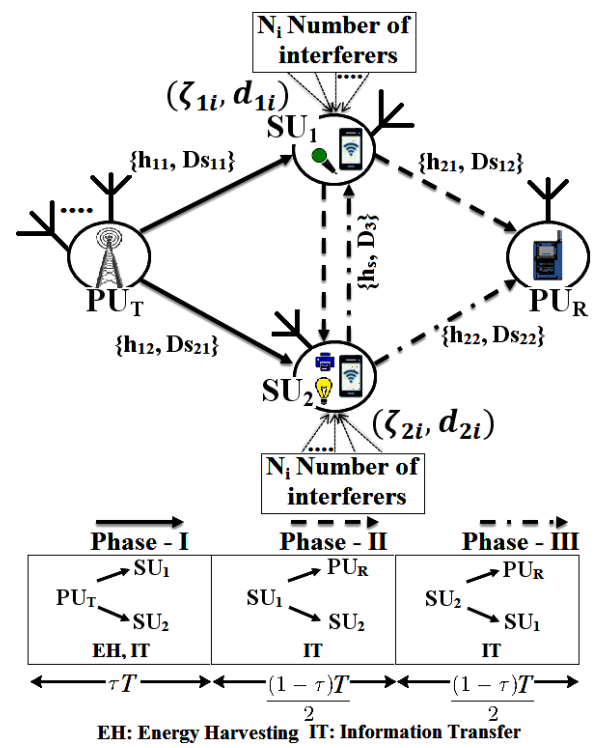

Fig. 1 System model and time frame structure

this $\mathrm{RF}$ signal for $\mathrm{EH}$ and information decoding. Then $\mathrm{SU}_{1}\left(\mathrm{SU}_{2}\right)$ node relays $\mathrm{PU}_{T}$ 's signal to $\mathrm{PU}_{R}$ along with its own information $X_{s j}$ towards $\mathrm{SU}_{p}(j \neq p ; j, p \in$ 1,$2 ; p=2$ for, $l=2$ and $p=1$ for, $l=3$ ) in the second (third) phase using the energy harvested in the first phase of the transmission frame. The received signal in the first phase at $S U_{j}$ from $\mathrm{PU}_{T}$ and $N_{i}$ number of CCIs, can be written as

$Y_{S U_{j}}=\sum_{n=1}^{N_{p_{a}}} \sqrt{\frac{P_{T}}{\left(D s_{j 1}\right)^{\nu}}} h_{1 j, n} X_{p t}+\sum_{i=1}^{N_{i}} \sqrt{\frac{P_{I_{j i}}}{\left(d_{j i}\right)^{\nu}}} \zeta_{j i} X_{I_{j i}}+n_{s u}$

where $\mathrm{P}_{T}$ and $P_{I_{j i}}$ are the transmission power from $\mathrm{PU}_{T}$ to $\mathrm{SU}_{j}$ and $\mathrm{I}_{i}$ to $\mathrm{SU}_{j}$, respectively. $\nu$ indicates the path loss exponent.

Due to very low noise power, it is reasonable that the system associated with RF-EH circuit is unable to harvest the energy from the noise signal. Therefore, the harvested energy at $\mathrm{SU}_{j}$, using the linear $\mathrm{EH}$ model, is expressed as $E_{j}=\eta \rho_{j}\left(\frac{\sum_{n=1}^{N_{p_{a}} P_{T}\left|h_{1 j, n}\right|^{2}}}{\left(D s_{j 1}\right)^{\nu}}+\frac{\sum_{i=1}^{N_{i}} P_{I_{j i}}\left|\zeta_{j i}\right|^{2}}{\left(d_{j i}\right)^{\nu}}\right) \tau T$. $\rho_{j}\left(0<\rho_{j}<1\right)$ indicates the PS factor. Harvested power, used by $\mathrm{SU}_{j}$ for broadcasting the information in the $l^{\text {th }}$ $(l \in 2,3)$ phase, is determined as $P_{j h}^{l}=\frac{E_{j}}{\frac{(1-\tau) T}{2}}$, where $0<\eta<1$ indicates RF to DC energy conversion efficiency, $a=\frac{\eta \rho_{j} P_{T}}{\left(D s_{j 1}\right)^{\nu}} \frac{2 \tau}{(1-\tau)}, b=\frac{\eta \rho_{j} P_{I_{j i}}}{\left(d_{j i}\right)^{\nu}} \frac{2 \tau}{(1-\tau)}$.

After EH, the remaining portion $\left(1-\rho_{j}\right)$ of the received power at $\mathrm{SU}_{j}$ is used to decode the information received during the time interval $\tau T$. The achievable rate of the information decoding in the first phase at 
$\mathrm{SU}_{j}$ can then be expressed as

$R_{S U_{j}}^{(I P)}=\frac{\tau T}{T} \log _{2}\left(1+\frac{\sum_{n=1}^{N_{p_{a}}} \frac{\left(1-\rho_{j}\right) P_{T}}{\left(D s_{j 1}\right)^{\nu}}\left|h_{1 j, n}\right|^{2}}{\frac{\sum_{i=1}^{N_{i}\left(1-\rho_{j}\right) P_{j i}\left|\zeta_{j i}\right|^{2}}}{\left(d_{j i}\right)^{\nu}}+\sigma^{2}}\right)$

Here $\beta(0<\beta<1)$ fraction of the total harvested power of $\mathrm{SU}_{j}$ is used to forward $\mathrm{PU}$ information and the remaining $(1-\beta)$ fraction of power is allocated to send the signal $X_{s j}$ to $\mathrm{SU}_{p}(j, p \in 1,2)$. Now, the combined signal transmission from $\mathrm{SU}_{j}$ is written as $X_{c s}=\sqrt{\beta P_{j h}^{l}} X_{p t}+\sqrt{(1-\beta) P_{j h}^{l}} X_{s j}$.

The received SINR $\gamma$, at $\mathrm{PU}_{R}$ in the $l^{\text {th }}$ phase, is written as $\gamma=\frac{a^{\prime} P_{j h}^{l}\left|h_{2 j}\right|^{2}}{b^{\prime} P_{j h}^{l}\left|h_{2 j}\right|^{2}+1}$ and $a^{\prime}=\frac{\beta}{\left(D s_{j 2}\right)^{\nu} \sigma^{2}}, b^{\prime}=$ $\frac{(1-\beta)}{\left(D s_{j 2}\right)^{\nu} \sigma^{2}}$.

The achievable rate at $\mathrm{PU}_{R}$ in the second phase is expressed as (based on the SINR at $\mathrm{PU}_{R}$ )

$R_{P U_{R j}}=\frac{(1-\tau)}{2} \log _{2}\{1+\gamma\}$

The received signal at $\mathrm{SU}_{p}$ is expressed as

$$
\begin{gathered}
Y_{S U_{p}}^{l}=\underbrace{\sqrt{\frac{(1-\beta) P_{j h}^{l}}{D_{3}^{\nu}}} h_{s} X_{s j}}_{\text {Desired signal }}+\underbrace{\sqrt{\frac{\beta P_{j h}^{l}}{D_{3}{ }^{\nu}}} h_{s} X_{p t}}_{\text {PU interfering signal }}+ \\
\underbrace{\sum_{i=1}^{N_{i}} \sqrt{\frac{P_{I_{p i}}}{\left(d_{p i}\right)^{\nu}}} \zeta_{p i} X_{I_{p i}}}_{\text {Interfering signals from interferers }}+\underbrace{n_{s u}}_{\text {Noise }}
\end{gathered}
$$

Based on the knowledge of the previous received information from $\mathrm{PU}$ signal, $\mathrm{SU}_{p}$ extracts the original information after canceling $\mathrm{PU}$ interfering component from the desired signal. Effectively, the achievable rate at $\mathrm{SU}_{p}$ is expressed as

$R_{S U_{R s_{p}}}=\frac{(1-\tau)}{2} \log _{2}\left\{1+\frac{c^{\prime} P_{j h}^{l}\left|h_{s}\right|^{2}}{\sum_{i=1}^{N_{i}} d i^{\prime}\left|\zeta_{p i}\right|^{2}+1}\right\}$

where $c^{\prime}=\frac{(1-\beta)}{\left(D_{3}\right)^{\nu} \sigma^{2}}, d i^{\prime}=\frac{P_{I_{p i}}}{\left(d_{p i}\right)^{\nu} \sigma^{2}}$.

\section{Outage Probability Analysis}

\subsection{PU Outage Probability}

Mathematically, PU outage probability is expressed as

$$
\mathscr{P}_{\text {out }}^{P U}=\left(1-\mathscr{P}_{s r_{1}}\right) \times\left(1-\mathscr{P}_{s r_{2}}\right)
$$

$$
\begin{aligned}
& \text { where } \mathscr{P}_{s r_{j}}=\quad \underbrace{\mathscr{P}\left(R_{S U_{j}}^{(I P)} \geq \mathscr{R}_{p}\right)} \\
& \underbrace{}_{\text {Success of link } \mathbf{P U}_{T} \rightarrow \mathbf{S U}_{j}} \\
& \times \underbrace{\mathscr{P}\left(R_{P U_{R j}} \geq \mathscr{R}_{p}\right)}_{\text {Success of link } \mathbf{S U} \mathbf{U}_{j} \rightarrow \mathbf{P} \mathbf{U}_{R}} .
\end{aligned}
$$

Using (2), success probability of data transmission from $\mathrm{PU}_{T}$ to $\mathrm{SU}_{j}$, can be expressed as

$$
\mathscr{P}\left\{\frac{A_{p_{j}} X_{j}}{C_{p_{j i}} Z_{j}+1} \geq u_{t 1}\right\}=\int_{\frac{u_{t 1}}{A_{p_{j}}}}^{\infty} f_{x_{j}}\left(x_{j}\right) d x_{j} \int_{0}^{\frac{A_{p_{j} x_{j}-u_{t 1}}}{{ }_{p_{j i}{ }^{u}{ }_{t 1}}}} f_{z_{j}}\left(z_{j}\right) d z_{j}
$$

where $A_{p_{j}}=\frac{\left(1-\rho_{j}\right) P_{T}}{\left(D s_{j 1}\right)^{\nu} \sigma^{2}}, C_{p_{j i}}=\frac{\left(1-\rho_{j}\right) P_{I_{j i}}}{\left(d_{j i}\right)^{\nu} \sigma^{2}}, u_{t 1}=2^{\left(\frac{\mathscr{R}_{p}}{\tau}\right)}-1$. Random variables $X_{j}$

$=\left|h_{1 j, n}\right|^{2}, X_{3}=\left|h_{2 j}\right|^{2}$ and $X_{s}=\left|h_{s}\right|^{2}$ follow Nakagami distribution. For mathematical simplicity, the power distribution of all the nearby interferers are considered to be equal. Here, $Z_{j}, V_{p}$ are the sum of $N_{i}$ number of independent identically distributed (i.i.d.) exponential random variables. They follow the central chisquared distribution given by $Z_{j}=\left|\zeta_{j i}\right|^{2}$ and $V_{p}=\left|\zeta_{p i}\right|^{2}$. Probability density function (PDF) of gamma distribution of $Z_{j}$ is expressed as $f_{Z_{j}}\left(z_{j}\right)=\frac{N_{i}^{N_{i}} z_{j}^{N_{i}-1} \exp \left(-\frac{z_{j}}{\frac{1}{N_{i}}}\right)}{\Gamma\left(N_{i}\right)}$. $\mathrm{PDF}$ of $X_{j}$ is expressed as

$f_{X_{j}}\left(x_{j}\right)=\frac{\left(N_{p_{a}} m_{k}\right)^{N_{p_{a}} m_{k}} x_{j}^{N_{p_{a}} m_{k}-1} \exp \left(-\frac{x_{j}}{N_{p_{a} m_{k}}}\right)}{\Gamma\left(N_{p_{a}} m_{k}\right)}$.

Therefore, (7) can be expressed as [11],

$$
\begin{array}{r}
\mathscr{P}\left\{\frac{A_{p_{j}} X_{j}}{C_{p_{j i}} Z_{j}+1} \geq u_{t 1}\right\}=\frac{\Gamma\left(N_{p_{a}} m_{k}, N_{p_{a}} m_{k} \frac{u_{t 1}}{A_{p_{j}}}\right)}{\Gamma\left(N_{p_{a}} m_{k}\right)}-\exp \left(\frac{N_{i} m_{k}}{C_{p_{j i}}}\right) \\
\frac{M_{p}\left(A_{p_{j}}\right)^{p_{a}-r}\left(u_{t 1}\right)^{r}\left(\frac{N_{i} m_{k}}{C_{p_{j i} u_{t 1}}}\right)^{p_{a}} \Gamma\left[m_{r n}, \frac{u_{t 1}}{A_{p_{j}}}\left(N_{p_{a}} m_{k}+\frac{N_{i} m_{k} A_{p_{j}}}{C_{p_{j i} u_{t 1}}}\right)\right]}{\left(N_{p_{a}} m_{k}+\frac{N_{i} m_{k} A_{p_{j}}}{C_{p_{j i} u_{t 1}}}\right)^{m_{r n}}}
\end{array}
$$

where $m_{r n}=N_{p_{a}} m_{k}+p_{a}-r, \Gamma(.,$.$) and \gamma(.,$.$) are the$ upper and the lower incomplete gamma function, respectively, $M_{p}=\frac{\sum_{p_{a}=0}^{N_{i} m_{k}-1} \sum_{r=0}^{p_{a}}(-1)^{r}\left(\begin{array}{c}p_{a} \\ r\end{array}\right)\left(N_{p_{a}} m_{k}\right)^{N_{p_{a}} m_{k}}}{p_{a} ! \Gamma\left(N_{p_{a}} m_{k}\right)}$.

Success probability of data transmission over the link $\mathrm{SU}_{j} \rightarrow \mathrm{PU}_{R}$ is defined as

$\left\{\begin{array}{l}\mathscr{P}\left[x_{3} \geq \frac{u_{t p}}{\left(a x_{j}+b z_{j}\right)}\right]=I, \quad \text { for }, u_{t 2}<\frac{\beta}{(1-\beta)} \\ 0, \quad \text { otherwise. }\end{array}\right.$

Now, $\mathscr{P}\left[x_{3} \geq \frac{u_{t p}}{\left(a x_{j}+b z_{j}\right)}\right]$ can be expressed as (10) (shown at the top of this page) [11]. Here $u_{t 2}=2^{\left(\frac{2 \mathscr{R}_{p}}{1-\tau}\right)}-$ $1, u_{t p}=\frac{u_{t 2}}{\left(a^{\prime}-b^{\prime} u_{t 2}\right)}, m_{x}=m_{k}-p_{a}+r$. The closed form expression of PU outage can be written as (8)-(10).

\subsection{SU Outage Probability}

Mathematically, SU outage probability can be expressed as $\mathscr{P}_{\text {out }}^{S U}=1-\left[\mathscr{P}\left(R_{S U_{1}}^{(I P)} \geq \mathscr{R}_{p}\right) \times \mathscr{P}\left(R_{S U_{2}}^{(I P)} \geq \mathscr{R}_{p}\right) \times\right.$ $\left.\mathscr{P}\left(R_{S U_{R s_{1}}} \geq \mathscr{R}_{s}\right) \times \mathscr{P}\left(R_{S U_{R_{2}}} \geq \mathscr{R}_{s}\right)\right]$. 


$$
\begin{aligned}
& I=\frac{M_{p}\left[2\left\{\frac{u_{t p} N_{i}}{b}\right\}^{\frac{\left(m_{x}\right)}{2}} K_{m_{x}}\left\{2 m_{k} \sqrt{\frac{u_{t p} N_{i}}{b}}\right\}-\frac{\left.2 \sum_{j_{t}=0}^{N_{p_{a}} m_{k}+r-1}\left\{\frac{u_{t p} N_{p_{a}}}{a}\right\}^{\frac{\left(m_{x}-j_{t}\right)}{2}}\left\{u_{t p} m_{k}\left(N_{p_{a}}-\frac{a N_{i}}{b}\right)\right\}^{j_{t}} K_{m_{x}-j_{t}}\left\{2 m_{k} \sqrt{\frac{u_{t p} N_{p_{a}}}{a}}\right\}\right]}{j_{t} ! a_{t}^{j}}\right]}{\Gamma\left(m_{k}\right)\left(\frac{1}{m_{k}}\right)^{m_{k}}\left(N_{p_{a}} m_{k}-\frac{a N_{i} m_{k}}{b}\right)^{N_{p_{a}} m_{k}+r}} \\
& \times\left(\frac{N_{i} m_{k}}{b}\right)^{p_{a}}\left(u_{t p}\right)^{p_{a}-r}(a)^{r} \Gamma\left(N_{p_{a}} m_{k}+r\right)+\left[\frac{2 \sum_{p_{a}=0}^{N_{p_{a}} m_{k}-1}\left(\frac{u_{t p} N_{p_{a}}}{a}\right)^{\frac{\left(m_{k}+p_{a}\right)}{2}}\left(m_{k}\right)^{m_{k}+p_{a}} K_{m_{k}-p_{a}}\left(2 m_{k} \sqrt{\frac{u_{t p^{N} p_{a}}}{a}}\right)}{p_{a} ! \Gamma\left(m_{k}\right)}\right]
\end{aligned}
$$

Similar to (10), $\mathscr{P}\left[\frac{c^{\prime}\left(a x_{j}+b z_{j}\right) x_{s}}{d i^{\prime} v_{p}+1} \geq u_{t s}\right]=I^{\prime}$ can be written as (11) (shown at the top of the next page) [11]. $u_{t s}=2^{\left(\frac{2 \mathscr{R}_{s}}{1-\tau}\right)}-1, m_{s r}=m_{k}+p_{a}-r_{b}+r_{g}-r_{c}-r_{m}$, $K_{\varrho}($.$) is modified Bessel's function. One can find the$ closed form expression of SU outage as in (8) and (11).

\section{Numerical Results}

The necessary simulation parameters are set as follows: noise variance $=-90 \mathrm{dBm}, \mathscr{R}_{p}=1 \mathrm{bps} / \mathrm{Hz}, \mathscr{R}_{s}=0.2 \mathrm{bps} / \mathrm{Hz}$, $L_{p}=70 \mathrm{~m}, d_{3}=15 \mathrm{~m}, \nu=2.7, \eta=0.9, \rho_{1}=\rho_{2}=\rho=0.9, \tau=0.5$. Both $\mathrm{SU}_{1}$ and $\mathrm{SU}_{2}$ are placed at the middle position between the two $\mathrm{PU}$ nodes. The received power at $\mathrm{SU}_{j}$ from $\mathrm{PU}_{T}$ is $1.5 \mathrm{dBm}$. An almost $10 \%$ value in PU outage probability is achieved at $\beta=0.94$ for $\mathrm{PU}$ transmission power $\left(\mathrm{P}_{T}\right)=43 \mathrm{dBm}$.

Figure 2.a illustrates both PU and SU outage performance with respect to the received INR at $\mathrm{SU}_{j}$. The simulation results match the analytical results. When INR value is small, both PU and SU outage are low. If INR value increases the outage performance of SU deteriorates (increases) rapidly and after a certain value of INR, the outage is found to be 1 . The PU outage improves (decreases) very slowly with the increasing value of INR and reaches the minimum value for a specific value of INR. Further enhancement in INR value leads to the deterioration of PU outage. This graphical plot shows the dual impact of CCI. Typical value of $20 \%$ and $15 \% \mathrm{PU}$ and SU outage performance gains are achieved for the increasing value of $N_{p_{a}}$ from 1 to 2 at $\mathrm{INR}=46$ $\mathrm{dB}$. If INR value increases beyond $50 \mathrm{~dB}, \mathrm{SU}$ outage improvement is insignificant for the increasing value of $N_{p_{a}}$ from 1 to 2 .

Figure 2.b.a and 2.c depict performances of both $\mathrm{PU}$ and SU outage as the variation on the transmission power of $\mathrm{PU}_{T}$ for different values of both $N_{i}, \rho$ and for different values of both $N_{p_{a}}, N_{i}$, respectively. Performance results are shown for the given value of the received $\mathrm{INR}=51 \mathrm{~dB}$. As $\beta$ is very high, PU outage performance is found to be better (low) than SU outage as seen in the results. Graphical results also show that performance is improved with the increasing value of $N_{p_{a}}$. As the interfering signal is another source of RF$\mathrm{EH}$, the amount of $\mathrm{EH}$ is increased with the increasing number of interferers and its impact is also reflected on the system performance. The change of PU outage is insignificant and SU outage is sensitive with the variation of $\mathrm{N}_{i}$ value. Due to this reason, PU outage variation is not shown for the corresponding change in $N_{i}$ value. Based on the graphical plot, it is found that if the value of $\rho$ is adjusted for a given value of interference power level, then both PU and SU outage gains are achieved. From Figure 2.b it is seen that almost $58 \%$ and $18 \%$ PU and SU outage performance gains are achieved at $\rho=0.9$ compared to $\rho=0.6$ at $N_{i}=4$ and $P_{T}=45 \mathrm{dBm}$. For the same average interference power, if the number of antenna changes from $N_{i}=1$ to 4 , then $11 \% \mathrm{SU}$ outage is found to be deteriorated.

Outage performance is significantly improved with the increasing number of antenna and is highlighted in Figure 2.c. Performance gains of $48 \%$ and $10 \%$ on $\mathrm{PU}$ and SU outage are found with the antenna number $N_{p_{a}}=3$ over $N_{p_{a}}=1$ for $N_{i}=4$ and $P_{T}=45 \mathrm{dBm}$. Furthermore, PU outage is found to be same and SU outage performance is found to be poor (high) in presence of CCI compared to its absence. The comparative study on SU outage performance between the proposed system with CCI and without CCI is shown in Table I.

Table 1 Comparative study of SU outage performance between presence of CCI and absence of CCI

\begin{tabular}{ccc}
\hline $\begin{array}{c}\text { Outage at transmit } \\
\text { power }=45 \mathrm{dBm}\end{array}$ & With CCI & Without CCI \\
\hline For $\rho_{1}=\rho_{2}=\rho=0.9$ & $0.5982\left(N_{i}=1\right)$, & $1.98 \times 10^{-10}$ \\
& $0.6724\left(N_{i}=4\right)$ & \\
\hline Improvement at & & \\
$\rho=0.9$ over $\rho=0.6$ & $18 \%\left(\right.$ for $\left.N_{i}=1\right)$ & $29 \%$ \\
\hline For $N_{p_{a}}=3$ & $0.65\left(N_{i}=4\right)$ & $1.78 \times 10^{-10}$ \\
\hline
\end{tabular}




$$
\begin{aligned}
& I^{\prime}=\frac{M_{p}\left(\frac{N_{i} m_{k}}{b}\right)^{p_{a}}\left(\frac{u_{t s}}{c^{c}}\right)^{p_{a}-r}(a)^{r} Y_{m}\left(d i^{\prime}\right)^{t_{u}-N_{i} m_{k}-t_{r}-r_{l}}\left(\frac{u_{t s}}{c^{b} b}\right)^{t_{u}-T_{u}} 2\left(\frac{a}{b}\right)^{t_{s}-t_{a}}(-a)^{r_{l}}\left(\frac{1}{b}\right)^{t_{a}-t_{r}-r_{l}}\left(\frac{u_{t s}}{c^{c}}\right)^{t_{a}-t_{r}-2 r_{l}}\left(\frac{N_{i} m_{k}}{d i^{t}}\right)^{r_{s}-r_{j}}}{\Gamma\left(m_{k}\right) \Gamma\left(N_{i} m_{k}\right)\left(N_{i} m_{k}\right)^{p_{a}-r-t_{k}-t_{s}}\left(N_{p_{a}} m_{k}\right)^{R_{t}-r_{t}}\left(\frac{N_{i} m_{k} a c^{\prime}}{u_{t s} d i^{\prime}}\right)^{r_{t}}} \\
& \left(m_{k}\right)^{m_{k}}\left(\frac{N_{p_{a}} m_{k} u_{t s}}{a c^{\prime}}\right)^{r_{j}}\left\{\frac{u_{t s} N_{p_{a}}}{a c^{c^{\prime}}}\right\}^{\frac{T_{m s}}{2}} K_{T_{m s}}\left\{2 m_{k} \sqrt{\frac{u_{t s} N_{p_{a}}}{a c^{p^{\prime}}}}\right\}+\left[1-2 \sum_{p_{a}=0}^{N_{i} m_{k}-1}\left(\frac{N_{i} m_{k}}{d i^{\prime}}\right)^{p_{a}-r_{g}} \sum_{r_{b}=0}^{p_{a}=0}(-1)^{r_{b}}\left(\frac{a c^{\prime}}{u_{t s}}\right)^{p_{a}-r_{b}-r_{m}}\right. \\
& \left.\frac{\left(d i^{\prime}\right)^{r_{m}} \Gamma\left(m_{r b}\right)\left(\frac{u_{t s} N_{p_{a}} m_{k}}{a c^{\prime}}\right)^{r_{c}-r_{g}} \sum_{r_{c}=0}^{m_{r b}-1} \sum_{r_{g}=0}^{r_{c}}\left(\begin{array}{l}
r_{g} \\
r_{g}
\end{array}\right)\left(\frac{u_{t s} N_{p_{a}}}{a c^{c}}\right)^{\frac{\left(m_{s r}\right)}{2}} K_{m_{s r}}\left(2 m_{k} \sqrt{\frac{u_{t s} N_{p_{a}}}{a c^{\prime}}}\right)}{r_{b} ! r_{c} ! \Gamma\left(m_{k}\right) \Gamma\left(N_{p_{a}} m_{k}\right)\left(\frac{1}{m_{k}}\right)^{m_{k}} \sum_{r_{m}=0}^{m_{r b}}\left(\frac{a c^{\prime}}{u_{t s} d i^{\prime}}\right)^{r_{m}}\left(\begin{array}{c}
m_{r b} b \\
r_{m}
\end{array}\right)\left(N_{p_{a}} m_{k}\right)^{p_{a}-r_{b}-r_{m}}}\right]
\end{aligned}
$$

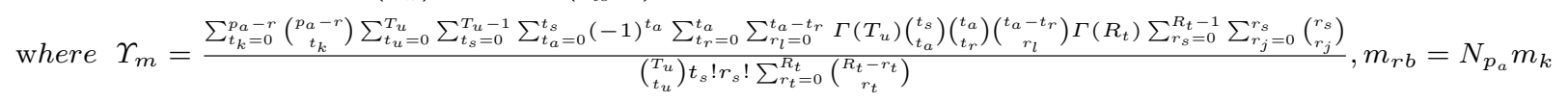

$$
\begin{aligned}
& +p_{a}-r_{b}, R_{t}=N_{p_{a}} m_{k}+r+t_{s}-t_{a}+r_{l}, T_{m s}=m_{k}-p_{a}+r+2 r_{l}+t_{r}-t_{a}-r_{j}-r_{t}, T_{u}=p_{a}-r-t_{k}+N_{i} m_{k} .
\end{aligned}
$$

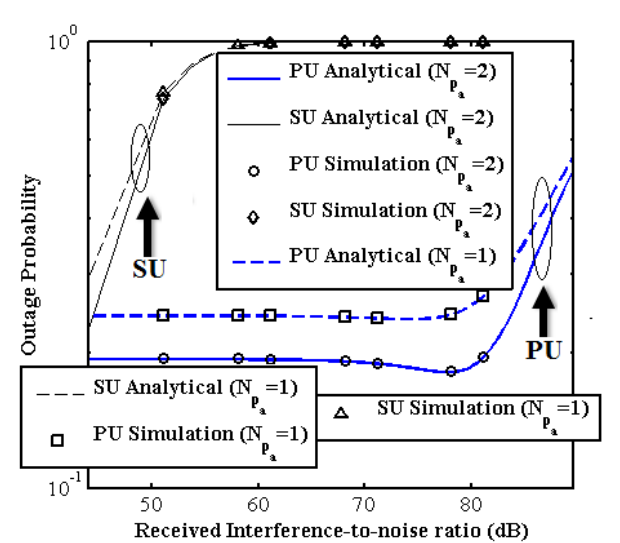

(a)

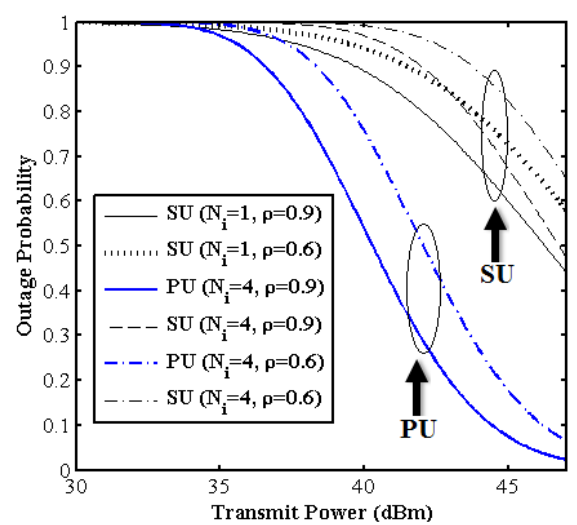

(b)

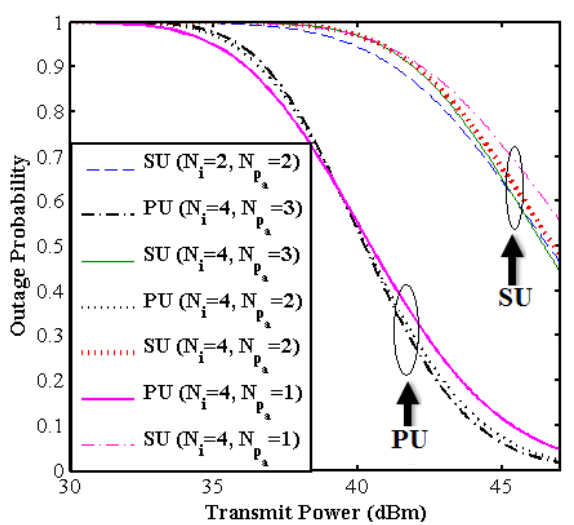

(c)

Fig. 2 Outage probability vs. (a) received INR at $\mathrm{SU}_{j}$ for $N_{i}=1$, (b) transmit power for different values of both $N_{i}$ and $\rho$ for $N_{p_{a}}=2$, (c) transmit power for different values of $N_{p_{a}}$ and $N_{i}$

\section{Conclusions}

In this paper, the effect of both CCI from multiple interferes and multi-antenna communication on the performance of SWIPT enabled one-way PU and two-way SU communications in overlay mode of CCRN is discussed. Closed form expressions of both PU and SU outages have been obtained that closely match the simulation results. Simulation results show improved outage performance at higher value of PS factor with an increase in number of CCI. It is also observed that with the increase in number of antenna from 1 to $3, \mathrm{PU}$ and $\mathrm{SU}$ outage performances are improved by $48 \%$ and $10 \%$, respectively.

\section{References}

[1 ] F. K. Ojo and M. F. Mohd Salleh, "Energy Efficiency Optimization for SWIPT-Enabled Cooperative Relay Networks in the Presence of Interfering Transmitter," IEEE Communications Letters, vol. 23, no. 10, pp. 1806-1810, Oct. 2019.
[2 ] I. Krikidis, S. Timotheou, S. Nikolaou, G. Zheng, D. W. K. Ng, R. Schober, "Simultaneous wireless information and power transfer in modern communication systems," IEEE Communications Magazine, vol. 52, no. 11, pp. 104 - 110, Nov. 2014.

[3 ] Y. Han, A. Pandharipande, and S. H. Ting, "Cooperative Decode-and-Forward Relaying for Secondary Spectrum Access," IEEE Transactions on Wireless Communications, vol. 8, no. 10, pp. 4945 - 4950, Oct. 2009.

[4 ] D. S. Gurjar, H. H. Nguyen and H. D. Tuan, "Wireless Information and Power Transfer for IoT Applications in Overlay Cognitive Radio Networks," IEEE Internet of Things Journal, vol. 6, no. 2, pp. 3257-3270, Apr. 2019.

[5 ] S. S. Kalamkar and A. Banerjee, "Interference-Aided Energy Harvesting: Cognitive Relaying With Multiple Primary Transceivers," IEEE Transactions on Cognitive Communications and Networking, vol. 3, no. 3, pp. 313327, Sep. 2017.

[6 ] Md. Fazlul Kader, "Interference aided cooperative SWIPT for cellular IoT networks towards $5 \mathrm{G}$ and beyond," Physical communication, vol. 43, pp. 1-18, Dec. 2020.

[7 ] J. He, S. Guo, G. Pan, Y. Yang and D. Liu, "Relay Cooperation and Outage Analysis in Cognitive Radio Networks With Energy Harvesting," IEEE Systems Journal, vol. 12, no. 3, pp. 2129-2140, Sep. 2018.

[8 ] S. Ghosh, T. Acharya, and S. P. Maity, "On Outage Analysis in SWIPT Enabled Bidirectional D2D Commu- 
nications using Spectrum Sharing in Cellular Networks," IEEE Transactions on Vehicular Technology, vol. 69, no. 9, pp. 10167 - 10176, Sep. 2020.

[9 ] S. T. Shah, K. W. Choi, S. F. Hasan, and M. Y. Chung, "Throughput analysis of two-way relay networks with wireless energy harvesting capabilities," Ad Hoc Networks, vol. 53, pp. 123 - 131, Dec. 2016.

[10 ] A. Prathima, D. S. Gurjar, H. H. Nguyen and A. Bhardwaj, "Performance Analysis and Optimization of Bidirectional Overlay Cognitive Radio Networks With HybridSWIPT," IEEE Transactions on Vehicular Technology, vol. 69, no. 11, pp. 13467-13481, Nov. 2020.

[11 ] I. S. Gradshteyn, and I. M. Ryzhik, "Table of Integrals, Series, and Products," Academic Press, Edition 7, 2007. 